\title{
Urinary Excretion Levels of MMX-Mesalazine as a Tool to Assess Non- Adherence
}

Tessa EH Romkens ${ }^{1,2 *}$, Jody Salomon ${ }^{1}$, Wilbert HM Peters ${ }^{1}$, David M Burger ${ }^{3}$, Frank Hoentjen ${ }^{1}$ and Joost PH Drenth ${ }^{1}$

${ }^{1}$ Department of Gastroenterology and Hepatology, Radboud university medical center, Nijmegen, The Netherlands

${ }^{2}$ Department of Gastroenterology and Hepatology, Jeroen Bosch Hospital, 's-Hertogenbosch, The Netherlands

${ }^{3}$ Department of Pharmacy and Radboud Institute for Health Sciences, Radboud university medical center, Nijmegen, The Netherlands

\begin{abstract}
Objective: 5-Amino salicylicacid (5-ASA) is the cornerstone of ulcerative colitis treatment, with (assessment of) non-adherence as a challenge. Multi-matrix release (MMX)-mesalazine has the advantage of once-daily (OD) dosing. Primarily we assessed urinary (NAc-) 5-ASA excretion, as measured by High-Performance Liquid Chromatography (HPLC), in order to monitor nonadherence, in healthy volunteers taking MMX-mesalazine. Secondly, we established urinary (NAc-)5-ASA cut-off levels for (partial) nonadherence.

Method: We studied 25 healthy adult volunteers who used MMX-mesalazine $2400 \mathrm{mg}$ OD (days 1-4), followed by $1200 \mathrm{mg}$ twice daily (BID) (days 8-11), separated by a drug-free interval of 3 days. Daily morning urine spot samples were collected. The cut-off level for adherence was set at the lowest steady state (NAc-)5-ASA urinary concentration level.

Results: Stability of urinary 5-ASA and NAc-5-ASA, stored at room temperature during 24 hours was $96.4 \pm 8.3 \%$ and $96.4 \pm$ $4.1 \%$. Recovery of urinary 5 -ASA and NAc-5-ASA was $114.3 \pm 10.4 \%$ and $107.5 \pm 6.4 \%$. The limit of detection and quantification were $1.1 \mathrm{ug} / \mathrm{ml}$ and $3.5 \mathrm{ug} / \mathrm{ml}$ for NAc-5-ASA and $0.4 \mathrm{ug} / \mathrm{ml}$ and $1.3 \mathrm{ug} / \mathrm{ml}$ for 5-ASA. The maximal 5-ASA within-run and between-run relative SD were $10.4 \%$ and $12.5 \%$. The cut-off level for non-adherence was determined at 9.67 (OD) and at 15.39 (BID) $\mathrm{mg} / \mathrm{mmol}$ (NAc-) 5-ASA per mmol creatinine.

Conclusion: HPLC is a feasible, sensitive and reproducible method to measure urinary (NAc-) 5-ASA excretion in volunteers taking MMX-mesalazine. This study establishes urinary (NAc-) 5-ASA cut-off levels for MMX-mesalazine non-adherence that may be useful in clinical practice and future trials.
\end{abstract}

Keywords: Inflammatory bowel disease; IBD; Adherence; Compliance; MMX-mesalazine; High-performance liquid chromatography (HPLC)

\section{Introduction}

Adherence is an important element for a successful treatment of patients with ulcerative colitis (UC). 5-Aminosalicylic acid (5-ASA) is a central component in UC treatment, but the reported prevalence of drug non-adherence is high and varies from 40 to $91 \%$ [1,5-6].

One of the reasons for this large variation is lack of direct and objective methods to screen for and monitor non-adherence in UC patients. Non-adherence puts patients at an increased risk for relapse [1-6], and steep incline in costs [7]. Already in 2006, a Cochrane review concluded that future trials with 5-ASA should focus on enhancement of patient's adherence [8], but assessing adherence has proven complex. Electronic monitoring using microelectronic chips that log date and time of medication bottle opening, is expensive and does not measure true intake of medication, but only opening of a bottle. On the other hand self-reporting measures are less reliable [9-12]. A more direct and objective way to measure adherence is to assess drug levels in biological fluids such as urine or plasma, and has been reliably measured by HighPerformance Liquid Chromatography (HPLC) [13-17]. A potential caveat of drug level monitoring at a clinical visit is the so called 'white coat compliance', which means that adherence tends to improve preceding a clinical visit $[11,18,19]$. Urinalysis is preferred here because of more stable drug metabolites that reflect medication use over a prolonged period of time. In 2003, Shale reported that $58 \%$ of patients with undetectable urinary drug levels admitted to be nonadherent [5]. Subsequent 5-ASA adherence studies using urinalysis applied different formulations, brands, and dosage frequencies. The novel once daily, high dosage ( $1.2 \mathrm{gr} / \mathrm{tablet}) \mathrm{MMX}$-mesalazine formulation has not yet been studied [20-22]. We now report a study to describe feasibility, sensitivity, and reproducibility of High-Performance Liquid
Chromatography (HPLC) to measure urinary (NAc-) 5-ASA excretion in healthy volunteers taking MMX-mesalazine. This allows us to determine cut-off levels for adherence, which can be used in future trials as well as in clinical practice.

\section{Method}

\section{Population}

This pilot study enrolled 25 healthy volunteers, who were studied for a total of 14 days. Volunteers were recruited via internet or billboard advertisements. Inclusion criteria were age between 18 and 80 years and use of adequate contraceptives during the study period. Pregnancy, significant co-morbidities or the use of co-medication (especially NSAIDs and drugs that possibly effect renal function) were exclusion criteria. After obtaining written informed consent, all volunteers were invited for baseline and screening visits by a research nurse. At baseline we obtained a brief medical history and a physical examination including urine spot and blood samples. Each volunteer received a trial number for identification purposes during the study. All study-related data were documented in a paper case report form (CRF) that was subsequently processed in a computerized database.

*Corresponding author: Tessa Romkens, Radboud university medical center Department of Gastroenterology and Hepatology, P.O. Box 9101, 6500 HB Nijmegen, The Netherlands, Tel: +31 243614760; Fax: +31 243540103; E-mail: tessa.romkens@radboudumc.nl

Received October 31, 2015; Accepted November 26, 2015; Published November 28, 2015

Citation: Romkens TEH, Salomon J, Peters WHM, Burger DM, Hoentjen F, et al. (2015) Urinary Excretion Levels of MMX-Mesalazine as a Tool to Assess NonAdherence. Pharm Anal Acta 6: 443. doi:10.4172/21532435.1000443

Copyright: @ 2015 Romkens T, et al. This is an open-access article distributed under the terms of the Creative Commons Attribution License, which permits unrestricted use, distribution, and reproduction in any medium, provided the original author and source are credited. 
Citation: Romkens TEH, Salomon J, Peters WHM, Burger DM, Hoentjen F, et al. (2015) Urinary Excretion Levels of MMX-Mesalazine as a Tool to Assess Non-Adherence. Pharm Anal Acta 6: 443. doi:10.4172/21532435.1000443

Page 2 of 6

\section{Study design}

The schematic outline of the study is described in Figure 1. Volunteers used $2400 \mathrm{mg}$ MMX-mesalazine once daily (OD) (2 tablets of $1200 \mathrm{mg}$ ), on study days 1 to 4 , and $1200 \mathrm{mg}$ twice daily (BID), on study days 8 to 11 . After day 4 and day 11, there was a drug-free washout interval of 3 days. OD medication was taken at breakfast (between 7 and 10 AM). BID medication was taken at breakfast (between 7 and $10 \mathrm{AM}$ ) and dinner (between 17 and $20 \mathrm{PM}$ ). The morning medication was taken under the watchful eye of the research nurse; the evening medication was either taken under nurse supervision, or assisted by webcam or smart-phone. At baseline and at study day 14 a blood sample was collected to analyze creatinine $(\mathrm{mmol} / \mathrm{l})$, hemoglobin, leucocytes, platelets, amylase, bilirubine, gamma-GT, and ASAT. The safety profile of MMX-mesalazine is similar to delayed-release mesalazine; it is recommended to check renal function at regular intervals [23-25]. Volunteers recorded adverse events through a purpose designed diary. All volunteers donated daily morning urine spot samples, which were frozen at $-20^{\circ} \mathrm{C}$, prior to HPLC analysis (samples collected before taking the morning dose of 5-ASA). In a previous study steady state urinary 5-ASA concentrations were reached after 48 hours [5]. Therefore mean individual urinary (NAc-) 5-ASA-excretion was determined on the individual steady state urinary values from day 3 to day 5 and day 10 to 12. The cut-off-level for adherence was defined as the lowest (NAc-) 5-ASA urinary concentration level found in the subjects, taking 2400 mg (OD or BID) MMX-mesalazine. All volunteers received a financial compensation (150 Euro) for their efforts. This trial (ISRCTN15765858) was approved by the Ethics Committee of Radboud university medical center, Nijmegen in the Netherlands.

\section{5-ASA medication}

MMX-mesalazine with MMX Multi Matrix System technology
(Mezavant ${ }^{\circledR}$; Shire Pharmaceuticals) is an oral, high-strength $1200 \mathrm{mg} /$ tablet, OD formulation of 5-ASA, that incorporates a gastro resistant, pH-dependent film coating combined with an MMX Multi Matrix System polymer core. The coating is thought to delay the release of 5-ASA during transit through the upper gastrointestinal tract, while hydrophilic and lipophilic excipients within the MMX tablet core are designed to prolong release of 5-ASA throughout the colon.

\section{High-performance Liquid chromatography (HPLC)}

Spot urine samples for further biochemical testing were frozen at $-20^{\circ} \mathrm{C}$ by the volunteers, transferred at room temperature and stored at $-20^{\circ} \mathrm{C}$ in the laboratory until further analysis. No difference in (NAc)5-ASA concentration was detected after 7 freeze-thawing cycles of urine samples (once freeze thawing: $54.0 \mathrm{mg} / \mathrm{mmol}$ creatinine; 7 times freeze thawing $54.1 \mathrm{mg} / \mathrm{mmol}$ creatinine). Samples were prepared by solvent extraction and by dilution technique. Routinely, at the start and end of each sequence calibration samples were used. These samples were prepared by adding 4 standard (NAc-) 5-ASA solutions to 0.05 $\mathrm{M}$ phosphate buffer ( $\mathrm{pH}$ 7.4). This was repeated after 42 samples. The reproducibility of our analyses was determined by repeating the test on the same urine sample every 30 samples. Urinary 5 -aminosalicylic acid (5-ASA) and N-acetyl-5-ASA (NAc-5-ASA) were measured by HPLC. The total 5-ASA concentration (5-ASA + NAc-5-ASA) is expressed as a ratio relative to the urinary creatinine excretion, to correct for variations in urinary concentration [5]. Urinary creatinine was measured using the Architect C16000 on basis of the Jaffe reaction [26], both obtained from Abbott Diagnostics (Lake Forest, Illinois, USA). Stock solutions of 5-aminosalicylic acid (Cayman Chemical Company, Ann Arbor, Michigan, USA), N-acetyl-5-aminosalicylic acid (Santa Cruz Biotechnology Inc, Santa Cruz, California, USA) and 4-aminosalicylic acid (Sigma-Aldrich, St. Louis, Missouri, USA) are

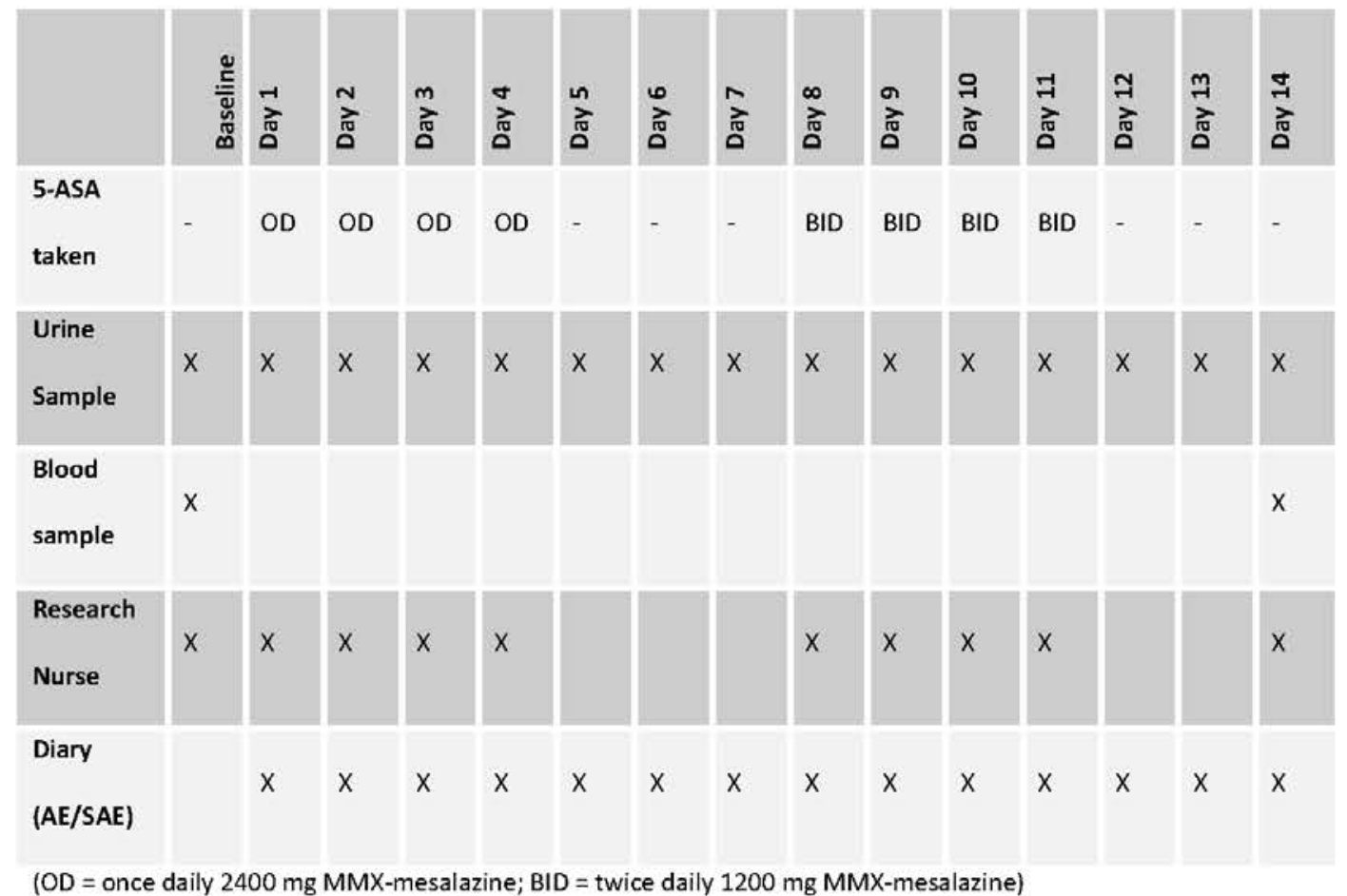

Figure 1: Schematic outline of the study. 
obtained by dissolving each compound in water, with a concentration of $100 \mu \mathrm{g} / \mathrm{ml}, 100 \mu \mathrm{g} / \mathrm{ml}$ and $6000 \mu \mathrm{g} / \mathrm{ml}$, respectively. These stock solutions are stored at $-80^{\circ} \mathrm{C}$ and are used to calibrate and standardize the assay. 5-ASA and NAc- 5-ASA are quantified by HPLC exactly as described by Hussain [13], using a Jasco HPLC system (JASCO, Tokyo, Japan) equipped with a PU-2089 plus pump, an AS-2055 plus auto sampler and a FP-2020 plus fluorescence detector (excitation 315 $\mathrm{nm}$, emission $430 \mathrm{~nm}$ ). Chromatographic separation of the eluent was attained using a supelcosil ABZ column ( $150 \mathrm{x} 4.6 \mathrm{~mm}$ I.D., 5 um silica particles) protected by a Supelco guard column ( $20 \mathrm{x} 4.6 \mathrm{~mm}$ I.D., $5 \mathrm{um}$ silica particles) both purchased from Sigma. The mobile phase consisted of $0.1 \mathrm{M}$ acetic acid, acetonitrile and triethylamine (1600:114:6, v/v/v) at $\mathrm{pH}$ 4.3. The flow-rate was $1.5 \mathrm{ml} / \mathrm{min}$. Sample preparation was performed following instruction of Hussain [13], with the exception of filtering the urine after centrifuging. The urine was diluted tenfold in $0.05 \mathrm{M}$ phosphate buffer ( $\mathrm{pH}$ 7.4). To a $0.1 \mathrm{ml}$ aliquot, $0.3 \mathrm{ml}$ of $0.05 \mathrm{M}$ phosphate buffer, $0.1 \mathrm{ml}$ of internal standard (4-ASA), $20 \mathrm{ul}$ propionic anhydride and $0.5 \mathrm{ml}$ of methanol was added.

The accuracy was assessed by spiking NAc-5-ASA and 5-ASA in blank matrices. The spiked samples were prepared in at least triplicate at three different concentrations.

The precision is based on the degree of repeatability of an analytical method under normal operation and is expressed as the percent relative standard deviation (RSD). To calculate the RSD 12 samples with known concentrations of NAc-5-ASA and 5-ASA were measured.

The limit of detection (LOD) and limit of quantification (LOQ) were determined by using the linear regression method. LOD is defined as the lowest concentration of (NAc-) 5-ASA in urine that can be detected. The LOD is expressed as a concentration at $3: 1, \mathrm{LOD}=3.3(\mathrm{SD} / \mathrm{S})$, where $S$ is the slope of the calibration curves. The limit of quantification (LOQ) is defined as the lowest concentration of (NAc-) 5-ASA in urine that can be determined with acceptable precision and accuracy. The LOQ is expressed as $\mathrm{LOQ}=10(\mathrm{SD} / \mathrm{S})$. Ten samples were measured to calculate the LOD and LOQ.

\section{Statistics}

The sample size of this small intervention study was determined at 25 , to the proposal of Julious with regard to pilot studies [27]. We used descriptive statistics for the characteristics of the tested cohort. All results were expressed as mean and SD or median and range values, unless otherwise indicated. Total urinary 5-ASA concentration (5-ASA and NAc-5-ASA) was expressed as a ratio to the urinary creatinine concentration, in mg $5-\mathrm{ASA} / \mathrm{mmol}$ creatinine. To determine the cutoff level of adherence as measured by total urinary 5-ASA excretion, we use the individual steady-state urinary 5-ASA-levels, for both dosing frequencies. These individual results are depicted in a scatter plot of urinary total 5-ASA to creatinine ratios. Each point in this plot represents one of the 25 individuals who took part in this study. To describe the reproducibility, variability and the range of urinary 5-ASA-excretion, the median (NAc-) 5-ASA/creatinine values of the total patient group are plotted in a column bar graph.

\section{Results}

\section{Demographics}

In a 4-month period 30 healthy volunteers were screened to participate in this pilot study. Four volunteers were excluded because of relevant co morbidity and co-medication. One volunteer started with the pilot study, but had to quit on day 6 , because of arthritis of the shoulder that necessitated NSAID treatment, which was an exclusion criterion. In total twenty-five volunteers were included and completed the whole study period of 14 days. Demographic variables of this study group and adverse events are presented in Table 1. Mean age of the volunteers was 38.1 years (SD 8.5); fifty two percent of the volunteers were male. Adherence was $100 \%$ assessed by nurse controlled medication intake.

\section{HPLC}

\section{Stability, accuracy, sensitivity and specificity}

Stability of 5-ASA and NAc-5-ASA in urine, stored at room temperature during 24 hours was $96.4 \pm 8.3 \%$ and $96.3 \pm 4.1 \%$, respectively. Stability data of urinary (NAc-) 5-ASA concentration at room temperature up to 192 hours are depicted in a Supplementary file (Figure 5a and 5b). No effect of 7 freeze-thawing cycles was detected on the concentrations of 5-ASA and NAc-5-ASA. The retention time for respectively NAc-5ASA, 5 ASA and 4-ASA is 6,11 and $16 \mathrm{~min}$. The accuracy or recovery of urinary 5-ASA and NAc-5-ASA was respectively $114.3 \pm 10.4 \%$ and $107.5 \pm 6.4 \%$. The linearity of the method was determined through analysis of five calibration curves containing non-zero concentrations.

The limit of detection (LOD) was $1.16 \mathrm{ug} / \mathrm{ml}$ for NAc-5-ASA and $0.43 \mathrm{ug} / \mathrm{ml}$ for 5 -ASA. The limit of quantification (LOQ) was respectively $3.51 \mathrm{ug} / \mathrm{ml}$ (NAc-5-ASA) and $1.29 \mathrm{ug} / \mathrm{ml}(5-\mathrm{ASA})$. The highest measured within-run relative standard deviation (R.S.D.) was respectively $4.1 \%$ for NAc-5-ASA, and $10.4 \%$ for 5 -ASA. The highest measured between-run R.S.D was $9.4 \%$ for NAc-5-ASA and $12.5 \%$ for 5-ASA.

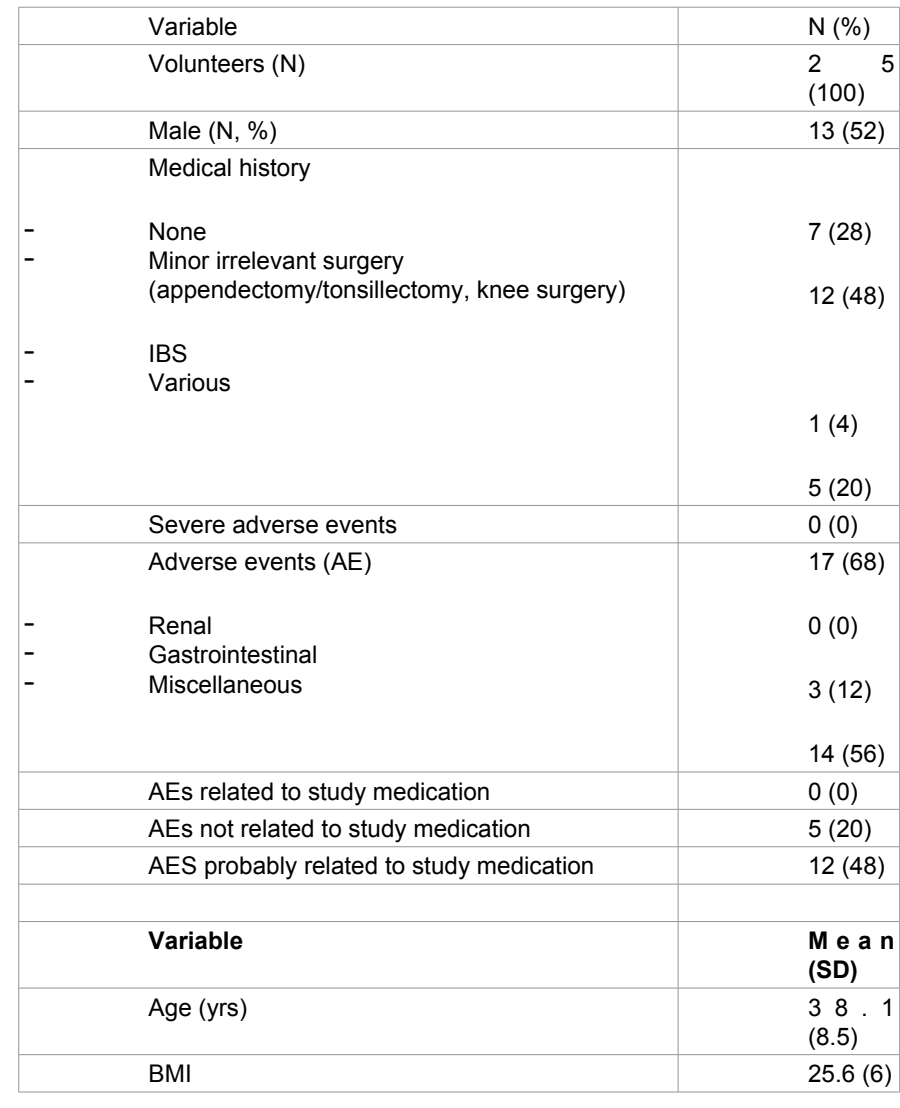

Table 1: Demographic variables and adverse events. 

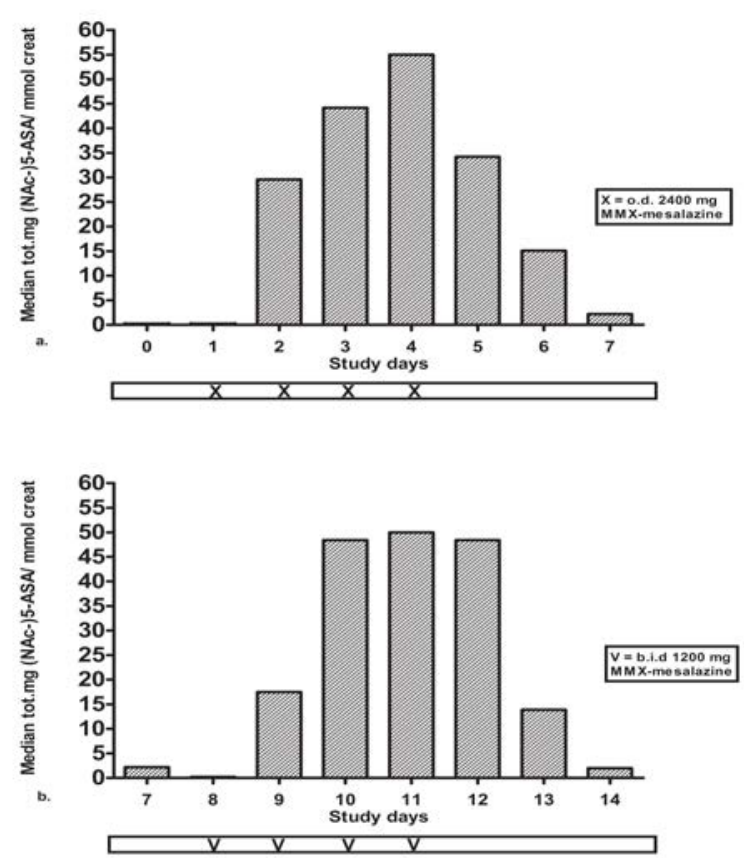

Figure 2: Mean individual steady state levels (NAc-)5-ASA/mmol creatinine. OD regime day 4-5 and BID regime day 11-12; --- cut-off levels for OD and BID regime

\section{Cut off level 5-ASA}

In Figure 3 the median (NAc-)5-ASA levels expressed as a ratio to the urinary creatinine concentration in the total patient group $(\mathrm{N}=25)$ are depicted. Steady state levels were respectively reached at day 4 and day 11 , and lasted until day 5 and day 12. Figure 4 shows mean individual steady state levels measured at day 4 to 5 , and at day 11 to 12. Cut-off level for adherence was defined as the lowest individual steady state (NAc-)5-ASA urinary concentration level found in the studied subjects. In the once daily medication schedule the cut-off level for adherence was $9.67 \mathrm{mg}$ (NAc-)5-ASA per mmol creatinine, and in the twice daily group this cut-off was determined at $15.39 \mathrm{mg}$ (NAc-) 5-ASA per mmol creatinine. Figure 1 shows individual data of the total patient group on day 6 ( 48 hours after last medication taken) and day 7 (72 hours after last medication taken). This figure provides insight into (partial) adherence of patients in daily practice, as the measured (NAc-)5-ASA urinary concentration level at day 6 is comparable to the value measured in a patient not taking his/her medication for two consecutive days. The lowest individual (NAc-)5-ASA urinary concentration level measured on day 6 is $0.03 \mathrm{mg}$ (NAc-) 5-ASA per mmol creatinine (median 15.1; range 0.03-66.24), and on day 7 this is $0.02 \mathrm{mg}$ (NAc-) 5-ASA per mmol creatinine (median 2.2; range 40.61).

\section{Adverse events}

During the pilot study no serious adverse events occurred. Adverse events probably related to the study medication occurred in $48 \%$ of the total group, and $12 \%$ complained of gastrointestinal symptoms (3/25). Routine laboratory measurements, particularly creatinine, remained unchanged during the complete study period (Table 2).

\section{Discussion}

This study documents the pharmacokinetics of a novel mesalazine formulation (MMX). We found that the use of HPLC for 5-ASA urinalysis in volunteers using MMX-mesalazine is feasible, reproducible, stable and sensitive, and is accompanied by a low interand intra-assay variation. This is in line with previously published studies, with other 5-ASA formulations, which showed that HPLC is a reliable tool to recover 5-ASA metabolites from urine samples [13-17].

There has been a paucity of pharmacokinetic data on MMXmesalazine. A literature search revealed published data derived from a single phase II clinical trial, the manufacturer-prescribing information, and an abstract published in 2007 [27-30]. These sources document a high pharmacokinetic variability among volunteers, comparable to our data in Figure 3 and 4 . They achieved steady state after 2 days, in contrast to our study (steady state achieved after 3 days in both dosing frequencies). We determined objective 5-ASA urinary cut-off values for (partial) non-adherence in our study that may be used in clinical practice or for the purpose of determination of adherence in clinical trials. Medication adherence occurs when the patient takes his/her medication according to the prescribed dosage, time, and frequency. Logically, if patients take no medication at all, they are absolute nonadherent, which corresponds to undetectable 5-ASA urinary levels. In practice, however, most patients occasionally forget to take their medication $[17,31]$, which defines them as partially non adherent. As such, it is important to provide objective 5-ASA urinary cut off values to define partial non-adherence.

A strong element of our study is the use of one single 5-ASA formulation, and the directly observed intake of MMX-mesalazine, which is efficacious in attaining complete adherence. A comprehensive review on this topic concluded that urinary 5-ASA excretion is comparable for many oral 5-ASA formulations, but that 5-ASA preparations with a foregut release profile demonstrate greater variability in urinary excretions. More recently released 5-ASA formulations, as MMX-mesalazine, were not included in this review [21].

Recent literature mentions a distinct variability in 5-ASA metabolism and distribution following oral dosing, and discovered an association between higher doses of oral mesalazine with higher serum concentrations and urinary excretion [32], a finding that was described before [5]. We defined cut off values for non-adherence for two different dosage schedules, which we consider an asset. We found a higher cut off level in the twice daily group $(15.39 \mathrm{mg} / \mathrm{mmol}$ (NAc-) 5-ASA per mmol creatinine) compared to the once daily group $(9.67$ $\mathrm{mg} / \mathrm{mmol}$ (NAc-) 5-ASA per mmol creatinine). Significance levels could not be determined because of the small sample size. Even though dosing frequency does not affect steady-state pharmacokinetics of delayed-release mesalazine, earlier studies found peak levels of 5-ASA serum concentrations in the early morning following divided daily dosing [32-34]. The indiscriminately use of different formulations, and dosage schedules may provide inaccurate values in defining a cut-off value in urinary 5-ASA excretion.

This study comes with some limitations. First of all, it is a small intervention study that carries the design of a pilot study. Secondly, we studied healthy volunteers instead of UC patients. A substantial part of the UC patients have co-morbidity or co-medication that may affect the pharmacokinetic properties of 5-ASA [30,35]. In patients with active UC, pharmacokinetics might be influenced by diarrhea, changed intestinal transit time, and luminal PH. The contribution of these elements and the ultimate effect on 5-ASA pharmacokinetics is incompletely understood and the literature reports conflicting results [21,36-40]. Interestingly, the pharmacokinetics of healthy volunteers 
Citation: Romkens TEH, Salomon J, Peters WHM, Burger DM, Hoentjen F, et al. (2015) Urinary Excretion Levels of MMX-Mesalazine as a Tool to Assess Non-Adherence. Pharm Anal Acta 6: 443. doi:10.4172/21532435.1000443

Page 5 of 6

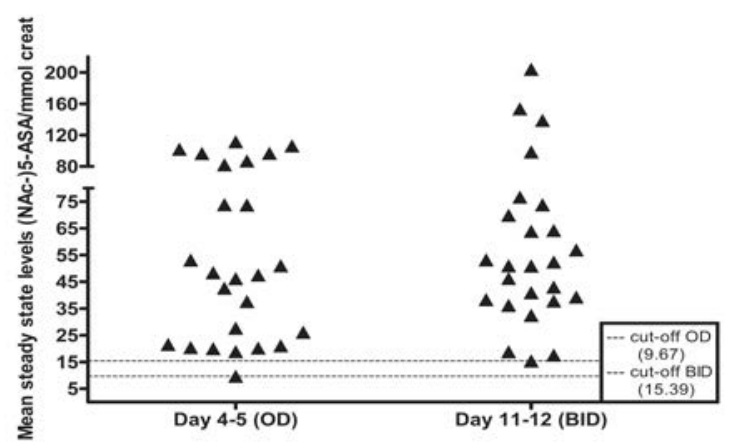

Figure 3: Individual (NAc-)5-ASA/mmol creatinine levels day 6 and day 7.

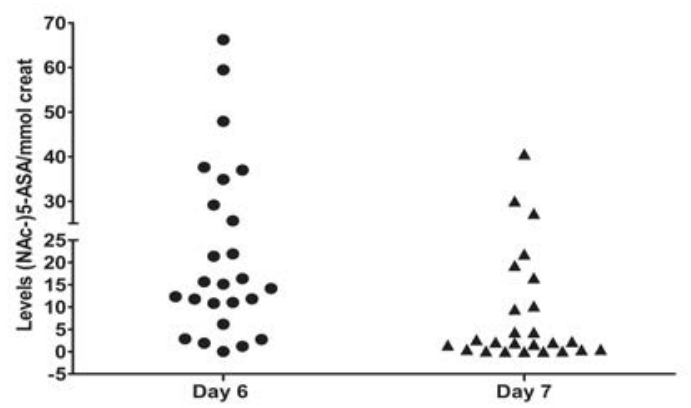

Figure 4: Stability data (NAc-)5-ASA at room temperature.

\begin{tabular}{|c|c|c|c|}
\hline Variable & $\begin{array}{c}\text { Visit day 1 } \\
\text { (mean, SD) }\end{array}$ & $\begin{array}{c}\text { Visit day 14 } \\
\text { (mean, SD) }\end{array}$ & p-value \\
\hline Creatinine (Umol/l) & $74.6(6.4)$ & $75.4(9.1)$ & NS \\
\hline Amylase (U/l) & $64.6(19.3)$ & $67.4(27.4)$ & NS \\
\hline Hemoglobin $(\mathrm{mmol} / \mathrm{l})$ & $8.9(0.6)$ & $9.0(0.7)$ & $\mathrm{NS}$ \\
\hline Thrombocytes $\left({ }^{*} 10^{9} / \mathrm{l}\right)$ & $244(50.7)$ & $260(61.1)$ & $\mathrm{NS}$ \\
\hline Leucocytes $\left({ }^{*} 10^{\circ} / \mathrm{l}\right)$ & $6.7(1.8)$ & $6.7(2.0)$ & $\mathrm{NS}$ \\
\hline Bilirubine $(\mathrm{Umol} / \mathrm{l})$ & $10.6(4.0)$ & $10.6(4.4)$ & $\mathrm{NS}$ \\
\hline Gamma-GT $(\mathrm{Umol} / \mathrm{l})$ & $27.6(15.0)$ & $28.5(16.4)$ & $\mathrm{NS}$ \\
\hline ASAT (U/l) & $24.4(4.7)$ & $27.1(8.6)$ & $\mathrm{NS}$ \\
\hline
\end{tabular}

Table 2: Laboratory results visit day 1 and 14 .

and patients with quiescent UC are comparable [40,41], and similar tissue concentrations of 5ASA and NAc-5ASA have been detected in both groups [13]. Therefore, in this specific subgroup of UC, data might be extrapolated. We did not perform formal plasma, $\mathrm{C}_{\max }$ analysis or AUC, which are accepted methods in pharmacokinetic studies. We specifically wanted to describe feasibility and reproducibility of HPLC in case of MMX-mesalazine users, and chose spot urinalysis because of the applicability of this method in daily practice. Of course, white coat compliance might affect results of spot urinalysis in the outpatient clinic. But, as described before $[5,42]$, and again shown in our study, it took more than three days without medication at all, to find near undetectable 5-ASA urine levels. For these reasons, we think this effect is negligible. Another matter of concern can be the costs of HPLC, which of course must be seen in the light of the costs of non-adherence.

In summary, HPLC spot 5-ASA urinalysis is fast, reproducible and sensitive. The cut off values for non-adherence in once or twice daily dosing are slightly different. Relevant for clinical practice is that we describe objective cut off values for (partial) non-adherence, which is the most common way of non-adherence. A spot 5-ASA urine sample below the described cut off values should trigger the treating physician to consider and discuss possible non-adherence.

\section{References}

1. Baars JE, Zelinkova Z, Mensink PB, Markus T, Looman CW, et al. (2009) High therapy adherence but substantial limitations to daily activities amongst members of the Dutch inflammatory bowel disease patients' organization: a patient empowerment study. Aliment Pharmacol Ther 30: 864-872.

2. Kane S, Huo D, Aikens J, Hanauer S (2003) Medication non-adherence and the outcomes of patients with quiescent ulcerative colitis. Am J Med 114: 39-43.

3. Kane SV (2006) Systematic review: adherence issues in the treatment of ulcerative colitis. Aliment Pharmacol Ther 23: 577-585.

4. Sewitch MJ, Abrahamowicz M, Barkun A, Bitton A, Wild GE, et al. (2003) Patient nonadherence to medication in inflammatory bowel disease. Am J Gastroenterol 98: 1535-1544

5. Shale MJ, Riley SA (2003) Studies of compliance with delayed-release mesalazine therapy in patients with inflammatory bowel disease. Aliment Pharmacol Ther 18: 191-198.

6. Kane SV, Cohen RD, Aikens JE, Hanauer SB (2001) Prevalence of nonadherence with maintenance mesalamine in quiescent ulcerative colitis. Am J Gastroenterol 96: 2929-2933.

7. Bassi A, Dodd S, Williamson P, Bodger K (2004) Cost of illness of inflammatory bowel disease in the UK: a single centre retrospective study. Gut 53: 14711478.

8. Sutherland L, Macdonald JK (2006) Oral 5-aminosalicylic acid for maintenance of remission in ulcerative colitis. Cochrane Database Syst Rev 10: CD000544.

9. Greenlaw SM, Yentzer BA, O'Neill JL, Balkrishnan R, Feldman SR (2010) Assessing adherence to dermatology treatments: a review of self-report and electronic measures. Skin Res Technol 16: 253-258.

10. Guerrero D, Rudd P, Bryant-Kosling C, Middleton B, Middleton BF (1993) Antihypertensive medication-taking. Investigation of a simple regimen. Am J Hypertens 6: 586-592.

11. Mihalko SL, Brenes GA, Farmer DF, Katula JA, Balkrishnan R, et al. (2004) Challenges and innovations in enhancing adherence. Control Clin Trials 25 447-457.

12. Osterberg L, Blaschke T (2005) Adherence to medication. N Engl J Med 353 : 487-497.

13. Hussain FN, Ajjan RA, Moustafa M, Anderson JC, Riley SA (1998) Simple method for the determination of 5-aminosalicylic and $\mathrm{N}$-acetyl-5-aminosalicylic acid in rectal tissue biopsies. J Chromatogr B Biomed Sci Appl 716: 257-266.

14. Fischer C, Maier K, Klotz U (1981) Simplified high-performance liquid chromatographic method for 5 -aminosalicylic acid in plasma and urine. Chromatogr 225: 498-503.

15. Tjørnelund J, Hansen SH (1991) High-performance liquid chromatographic assay of 5-aminosalicylic acid (5-ASA) and its metabolites N-beta-Dglucopyranosyl-5-ASA, N-acetyl-5-ASA, N-formyl-5-ASA and N-butyryl-5-ASA in biological fluids. J Chromatogr 570: 109-117.

16. Shaw IS, Jobson BA, Silverman D, Ford J, Hearing SD, et al. (2002) Is you patient taking the medicine? A simple assay to measure compliance with 5 -aminosalicylic acid-containing compounds. Aliment Pharmacol Ther 16 2053-2059.

17. Cervený $\mathrm{P}$ Bortlík M, Kubena A, Vlcek J, Lakatos PL, et al. (2007) Nonadherence in inflammatory bowel disease: results of factor analysis. Inflamm Bowel Dis 13: 1244-1249.

18. Feldman SR, Camacho FT, Krejci-Manwaring J, Carroll CL, Balkrishnan R (2007) Adherence to topical therapy increases around the time of office visits. J Am Acad Dermatol 57: 81-83.

19. Cramer JA, Scheyer RD, Mattson RH (1990) Compliance declines between clinic visits. Arch Intern Med 150: 1509-1510.

20. Moshkovska T, Stone MA, Clatworthy J, Smith RM, Bankart J, et al. (2009) An investigation of medication adherence to 5-aminosalicylic acid therapy in patients with ulcerative colitis, using self-report and urinary drug excretion measurements. Aliment Pharmacol Ther 30: 1118-1127. 
Citation: Romkens TEH, Salomon J, Peters WHM, Burger DM, Hoentjen F, et al. (2015) Urinary Excretion Levels of MMX-Mesalazine as a Tool to Assess Non-Adherence. Pharm Anal Acta 6: 443. doi:10.4172/21532435.1000443

21. Sandborn WJ, Hanauer SB (2003) Systematic review: the pharmacokinetic profiles of oral mesalazine formulations and mesalazine pro-drugs used in the management of ulcerative colitis. Aliment Pharmacol Ther 17: 29-42.

22. Hanauer SB (2004) Review article: aminosalicylates in inflammatory bowe disease. Aliment Pharmacol Ther 20 Suppl 4: 60-65.

23. Moum B (2008) Which are the 5-ASA compound side effects and how is it possible to avoid them? Inflammatory bowel diseases 2: S212-213.

24. Lichtenstein GR, Kamm MA, Boddu P, Gubergrits N, Lyne A, et al. (2007) Effect of once- or twice-daily MMX mesalamine (SPD476) for the induction of remission of mild to moderately active ulcerative colitis. Clin Gastroenterol Hepatol 5: 95-102.

25. D'Haens G, Sandborn WJ, Barrett K, Hodgson I, Streck P (2012) Once-daily $\operatorname{MMX}\left(\hat{A}^{\circledR}\right)$ mesalamine for endoscopic maintenance of remission of ulcerative colitis. Am J Gastroenterol 107: 1064-1077.

26. Husdan H, Rapoport A (1968) Estimation of creatinine by the Jaffe reaction. A comparison of three methods. Clin Chem 14: 222-238.

27. Julious SA (2005) Sample size of 12 per group rule of thumb for a pilot study. Pharmaceutical Statistics 4: 287-91.

28. D'Haens G, Hommes D, Engels L, Baert F, van der Waaij L, et al. (2006) Once daily MMX mesalazine for the treatment of mild-to-moderate ulcerative colitis: a phase II, dose-ranging study. Aliment Pharmacol Ther 24: 1087-1097.

29. Sandborn W, Balan G, Kuzmak B, Hanauer S (2007) Comparable pharmacokinetics of two delayed release formulations of oral mesalamine. Am J Gastroenterol 102.

30. Shire. US. inc. Lialda (mesalamine) delayed release tablets: US prescribing information.

31. Tomaszewski M, White C, Patel P, Masca N, Damani R, et al. (2014) High rates of non-adherence to antihypertensive treatment revealed by high-performance liquid chromatography-tandem mass spectrometry (HP LC-MS/MS) urine analysis. Heart 100: 855-861.

32. Hussain FN, Aijan RA, Riley SA (2000) Dose loading with delayed-release mesalazine: a study of tissue drug concentrations and standard pharmacokinetic parameters. Br J Clin Pharmacol 49: 323-330.
33. De Mey C, Meineke I (1992) Prandial and diurnal effects on the absorption of orally administered enteric coated 5-aminosalicylic acid (5-ASA). Br J Clin Pharmacol 33: 179-182.

34. Hussain FN, Aijan RA, Kapur K, Moustafa M, Riley SA (2001) Once versus divided daily dosing with delayed-release mesalazine: a study of tissue drug concentrations and standard pharmacokinetic parameters. Aliment Pharmaco Ther 15: 53-62.

35. Román AL, Muñoz $\mathrm{F}$ (2011) Comorbidity in inflammatory bowel disease. World J Gastroenterol 17: 2723-2733.

36. Fallingborg J, Christensen LA, Jacobsen BA, Rasmussen SN (1993) Very low intraluminal colonic $\mathrm{pH}$ in patients with active ulcerative colitis. Dig Dis Sci 38 1989-1993.

37. Reddy SN, Bazzocchi G, Chan S, Akashi K, Villanueva-Meyer J, et al. (1991) Colonic motility and transit in health and ulcerative colitis. Gastroenterology 101: 1289-1297.

38. Christensen LA, Slot O, Sanchez G, Boserup J, Rasmussen SN, et al. (1987) Release of 5-aminosalicylic acid from Pentasa during normal and accelerated intestinal transit time. Br J Clin Pharmacol 23: 365-369.

39. Evans DF, Pye G, Bramley R, Clark AG, Dyson TJ, et al. (1988) Measurement of gastrointestinal $\mathrm{pH}$ profiles in normal ambulant human subjects. Gut 29 1035-1041.

40. Mahmud N, Weir DG, Kelleher D (1999) Systemic levels of free 5-aminosalicylic acid depend on the nature of the 5-aminosalicyclic acid derivative and not on disease activity or extent in patients with inflammatory bowel disease. Ir J Med Sci 168: 228-232.

41. Norlander B, Gotthard R, Strom M (1991) Steady-state pharmacokinetics of enteric coated 5-amino-salicylic acid tablets in healthy volunteers and in patients with Crohn's disease or ulcerative colitis. Aliment Pharmacol Ther 5 291-300.

42. Gifford AE, Berg AH, Lahiff C, Cheifetz AS, Horowitz G, et al (2013) A random urine test can identify patients at risk of mesalamine non-adherence: a prospective study. Am J Gastroenterol 108: 249-255. 